\title{
BMJ Open Collaborating with healthcare providers to understand their perspectives on a hospital-to-home warning signs intervention for rural transitional care: protocol of a multimethod descriptive study
}

\author{
Mary T Fox (D) , ${ }^{1}$ Jeffrey I Butler (D) , ${ }^{1}$ Souraya Sidani (D) , ${ }^{2}$ Evelyne Durocher (D) , ${ }^{3}$ \\ Behdin Nowrouzi-Kia (D) , ${ }^{4}$ Janet Yamada, ${ }^{2}$ Sherry Dahlke (D) , ${ }^{5}$ Mark W Skinner (D) ${ }^{6}$
}

To cite: Fox MT, Butler Jl, Sidani S, et al. Collaborating with healthcare providers to understand their perspectives on a hospital-to-home warning signs intervention for rural transitional care: protocol of a multimethod descriptive study. BMJ Open 2020;10:e034698. doi:10.1136/ bmjopen-2019-034698

- Prepublication history and additional material for this paper are available online. To view these files, please visit the journal online (http://dx.doi. org/10.1136/bmjopen-2019034698).

Received 02 0ctober 2019 Revised 18 February 2020 Accepted 25 March 2020

Check for updates

(C) Author(s) (or their employer(s)) 2020. Re-use permitted under CC BY-NC. No commercial re-use. See rights and permissions. Published by BMJ.

For numbered affiliations see end of article.

Correspondence to

Dr Mary T Fox;

maryfox@yorku.ca

\section{ABSTRACT}

Introduction This study builds on our prior research, which identified that older rural patients and families (1) view preparation for detecting and responding to worsening health conditions as their most pressing unmet transitional care (TC) need and (2) perceive an evidencebased intervention, preparing them to detect and respond to warning signs of worsening health conditions, as highly likely to meet this need. Yet, what healthcare providers need to implement a warning signs intervention in rural TC is unclear. The objectives of this study are (1) to examine healthcare providers' perspectives on the acceptability of a warning signs intervention and (2) to identify barriers and facilitators to healthcare providers' provision of the intervention in rural communities.

Methods and analysis This multimethod descriptive study uses a community-based, participatory research approach. We will examine healthcare providers' perspectives on a warning signs intervention. A purposive, criterion-based sample of healthcare providers stratified by professional designation (three strata: nurses, physicians and allied healthcare professionals) in two regions (Southwestern and Northeastern Ontario, Canada) will (1) rate the acceptability of the intervention and (2) participate in small ( $\mathrm{n}=4-6$ healthcare providers), semistructured telephone focus group discussions on barriers and facilitators to delivering the intervention in rural communities. Two to three focus groups per stratum will be held in each region for a total of 12-18 focus groups. Data will be analysed using conventional qualitative content analysis and descriptive statistics. Ethics and dissemination Ethics approval was obtained from the Office of Research Ethics at York University and the Health Sciences North Research Ethics Board. Findings will be communicated through plain language summary and policy briefs, press releases, manuscripts and conferences.

\section{INTRODUCTION}

Preamble

Transitional care (TC) refers to the healthcare services patients receive as they move

\section{Strengths and limitations of this study}

- This study uses a systematic approach to assess multiple healthcare providers' perspectives on the acceptability of a warning signs intervention for rural transitional care.

- The use of telephone focus groups may enhance participation by mitigating logistical and geographical barriers (eg, long travel distance) but may also prevent the reading of facial expressions and body language, which in face-to face focus groups provide further insight into participants' opinions and group interactions.

- Because a focus group is a group event and anonymity cannot be guaranteed, some participants may be less forthcoming than in a 1:1 interview.

- Using multiple methods of recruitment, providing an incentive and describing, in detail, healthcare provider demographic and professional profiles are strategies to mitigate the potential for selection bias and enhance the generalisability of findings to similar settings beyond Ontario.

- Recruiting physicians may be a challenge due to their more limited availability than other healthcare providers in rural communities.

from hospital to home and includes timelimited interventions designed to optimise postdischarge care management. ${ }^{12}$ Patients in rural communities, defined as small towns outside the commuting zones of large urban centres with populations of $>100000,{ }^{3}$ have poorer TC outcomes than patients in urban communities. ${ }^{4}$ This study builds on our prior TC research and consultations with knowledge users (administrative and clinical decision-makers), in which we identified that (1) the most pressing unmet TC need of older rural medical patients at risk of readmission 
and their families is how to detect and respond to worsening health conditions; and (2) an evidence-based intervention (hereafter referred to as a warning signs intervention) was viewed as highly likely to meet this need ${ }^{5}$ However, how best to support healthcare providers (HCPs) in implementing this warning signs intervention in rural communities remains unclear. Filling this knowledge gap is vital to enable HCPs to deliver the intervention and to improve TC outcomes in rural communities.

\section{Overall goals}

The overall goals of this study are to address the knowledge gap on the implementation of a warning signs intervention and to provide high-quality, actionable evidence to optimise its implementation in rural communities.

\section{Rationale}

In many healthcare jurisdictions, hospital readmissions of older patient populations with increasingly complex health conditions have made TC a key priority. Nowhere is this need more urgent than in rural communities, where patients have significantly more emergency department visits and hospital readmissions during the 30-day postdischarge period than their urban counterparts. ${ }^{4}$ During that 30 -day period, almost $60 \%$ of rural patients visit the emergency department for a non-urgent health problem, and up to $59 \%$ of rural patients' hospital readmissions are preventable. ${ }^{4}$ These trends underscore inadequacies in preparing medical patients and their families on postdischarge care management and foreground the urgent need to improve TC in rural communities to address rural-urban disparities in TC effectiveness. Rural communities are characterised by lower socioeconomic status than urban communities and long-standing trends of health service deprivation (eg, fewer health system resources), as well as obstacles to availability and use of health services related to geography, leaving many people highly dependent on informal family care. ${ }^{6}$ Furthermore, rural communities have higher percentages of older people (aged 60+ years) with higher rates of multiple chronic conditions ( $\geq 2$ concurrent chronic conditions, such as respiratory and heart diseases $)^{7}$ than urban communities. ${ }^{8}$ Consequently, older rural medical patients typically require more complex care management, ${ }^{7}$ and their care requirements often change considerably postdischarge. $^{2}$ The transition from hospital to home marks a particularly pronounced shift from provider-driven to self-managed and family-managed care for rural patients and their families. Once home, many patients and their families must manage care that was previously provided by nurses in hospital, such as monitoring and responding to changes in health conditions, often with little or no training and professional support. ${ }^{29}$

In our prior study, older rural medical patients at risk of hospital readmission and their families identified knowing how to detect and respond to worsening health conditions as their most pressing unmet TC need. ${ }^{5}$ They described receiving minimal preparation in hospital or at home about how to detect and respond to worsening health conditions, and needing more information about whether a sign or symptom was 'normal' or cause for concern. These findings corroborate other research in Ontario highlighting that a lack of knowledge about warning signs is a pervasive problem; according to Health Quality of Ontario, $41 \%$ of patients discharged from Ontario hospitals do not know the signs or symptoms indicating that their health conditions may be worsening. ${ }^{10}$ These findings are especially troubling, given that older medical patients are increasingly discharged home 'sicker and quicker', ${ }^{11}$ are more vulnerable to breakdowns in the continuity of care during the transfer home ${ }^{12}$ and have the highest hospital readmission rates in rural Ontario $(65 \%) .{ }^{4}$ Consequently, these patients have a great need for TC emphasising self-management and family management of care and recovery. Consultations with knowledge users have confirmed the importance of strengthening the warning signs aspect of TC.

TC is initiated in hospital predominantly by nurses in collaboration with other HCPs. ${ }^{13}$ Most models of TC emphasise the importance of preparing patients to identify and respond to warning signs of worsening health conditions. $^{12}{ }^{14-18}$ Studies of TC trials have found a warning signs intervention, as part of multicomponent hospital-to-home TC programmes, to be effective in reducing emergency department visits ${ }^{19}{ }^{20}$ and hospital readmissions, ${ }^{13} 18$ 20-22 and in improving patient knowledge and confidence in managing changes in their health conditions $^{1}$ (all $\mathrm{p}<0.05$, small to moderate effect sizes).

Informed by a literature synthesis, we developed a comprehensive description of a warning signs intervention. ${ }^{5}$ The goal of this intervention is for patients and their families to know the signs and symptoms that may indicate patients' health conditions are worsening and what to do. ${ }^{5}$ The intervention starts in the hospital with an assessment of patients' and families' overall health literacy, what they already know about the warning signs specific to the patient's conditions, their ability to detect and respond to them at home, and what they need to learn to respond appropriately. ${ }^{5}$ The intervention is provided before and after discharge and, to enhance uptake, incorporates educational strategies including the 'teach-back' method, as well as written materials that use simple terms, symbols or pictograms to foster understanding of how to monitor health conditions and detect and respond to warning signs, such as when to get help, who to contact and when to go to the emergency department. ${ }^{5}$

In our previous study, older rural medical patients at risk of readmission and their families rated this warning signs intervention, using a validated tool, as 'very' to 'very much' acceptable (mean rating=3.28-3.7/4.0, respectively) in preparing them to detect and respond to worsening health conditions. ${ }^{5}$ Acceptability refers to the desirability of an intervention in addressing a health problem or need and is vital to intervention implementation by HCPs, uptake by patients and effectiveness. ${ }^{23} 24$ The Medical Research Council maintains that 
intervention implementation is frequently challenged by problems of acceptability that limit implementation and undermine effectiveness. ${ }^{25}$ Assessing the acceptability of interventions to HCPs is thus essential to understanding how to enhance intervention implementation because interventions perceived as acceptable are more likely to be provided than those perceived as unacceptable. ${ }^{23} 24$ However, HCPs' perspectives on the acceptability of this warning signs intervention, as well as the barriers and facilitators to its delivery, are unknown. Prior studies have identified barriers (eg, lack of training, ${ }^{26}$ limited experience $^{27}$ and poor communication ${ }^{28}$ ) and facilitators (eg, HCP buy-in ${ }^{29}$ and interprofessional collaboration ${ }^{27}{ }^{29}$ ) to TC in general, but they have not examined HCPs' perspectives on the specific, situated barriers and facilitators of a warning signs intervention in rural communities. Accordingly, this study builds on our previous research by examining HCPs' perspectives on this warning signs intervention for TC in rural communities. The study will provide actionable evidence on what HCPs need to implement the intervention.

\section{Study objectives}

The study objectives were to

1. Examine HCPs' perspectives on the acceptability of a warning signs intervention in TC with older rural medical patients at risk of readmission and their families.

2. Identify barriers and facilitators to HCPs' provision of the intervention.

\section{METHODS AND ANALYSIS \\ Design}

This multimethod descriptive study focuses on gathering HCPs' perspectives on a warning signs intervention using quantitative measures of intervention acceptability, followed by semistructured focus group questions exploring the intervention's acceptability in greater depth, as well as barriers and facilitators to its implementation

\section{Healthcare Providers}

\section{Target Population}

Nurses, physicians, and allied healthcare professionals in Southwestern and Northeastern

Ontario.

\section{Eligibility}

Assess for eligibility.

\section{Data Collection Method}

Online/hard copy to administer measures $\&$ telephone focus groups to ask semistructured questions.

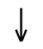

\section{Focus on}

Perceived acceptability of a Warning Signs intervention.

Barriers and facilitators to its delivery to older rural medical patients at risk for readmission and their families.

\section{Findings}

Assessment of intervention acceptability and identification of barriers and facilitators expected to influence intervention implementation.

\section{$\downarrow$}

In-depth understanding of healthcare providers' perspectives on the acceptability of a warning signs intervention.

List of barriers and facilitators to implementing a warning signs intervention, as identified by healthcare providers.

High-quality evidence for researchers, knowledge users, and policy makers to inform implementation of a warning signs intervention in rural communities. 
(figure 1). The study design is guided by the intervention acceptability $^{24}$ and knowledge-to-action ${ }^{30}$ frameworks. Both conceptual frameworks are rooted in community-based, participatory research and emphasise the importance of engaging HCPs when examining intervention acceptability and implementation. ${ }^{24}{ }^{30}$ The frameworks guide researchers in systematically assessing HCPs' perspectives on the acceptability of an intervention, as well as the barriers and facilitators to its delivery, in order to design a plan $^{23} 31$ that enables HCPs' implementation of the intervention with fidelity to maximise its effectiveness. ${ }^{24} 30$

\section{Setting and sample}

The study, which runs from November 2019 to November 2020, will be conducted in Ontario, which has a substantial rural population (more than 250000 Ontarians reside in rural communities) that is older than the national average. $^{32}$ Accordingly, the purposive, criterion-based sample of HCPs will be stratified by professional designation and region, as recommended by knowledge users on our team. The three strata are nurses, physicians and allied healthcare professionals. The two regions are Southwestern and Northeastern Ontario. The criteria for HCP inclusion are working $\geq 21$ hours $/$ week $^{33}$ in a Southwestern or Northeastern Ontario hospital and/or community care (eg, primary care) setting and providing TC to rural patients with medical conditions; these patients represented the majority of the sample in our prior research identifying the need to know how to detect and respond to warning signs. ${ }^{5}$

HCPs within each stratum will be invited to participate in separate focus group interviews. Homogenous focus groups will promote HCPs' comfort $^{34}$ and ensure that professional hierarchies do not prevent HCPs from expressing their perspectives. ${ }^{35}$ Given that some HCPs' work straddles both settings, the focus groups will include both HCPs who work in the hospital and those who work in community care.

\section{Sample size}

Two to three focus groups per each of the three strata, with 4-6 HCPs per group, will be held in each region, for a total of 6-9 focus groups per region and a total sample size of 48-108 HCPs. Focus groups will be conducted until informational saturation (the point in data collection when data become redundant and no new descriptive codes, categories or themes are being generated) ${ }^{36}$ is achieved, based on the rationale that a minimum of two focus groups per stratum is needed to achieve saturation. ${ }^{34}$ This number of focus groups falls within the range recommended for sufficient and feasible qualitative data collection and management. ${ }^{37}$ The total number of HCPs rating the acceptability of the intervention is sufficient to describe their scores on the Intervention Acceptability Scale and items. ${ }^{38}$

\section{Data collection}

\section{Quantitative measures}

Intervention acceptability will be examined using the Intervention Acceptability Scale. ${ }^{39} 40$ The scale has shown internal consistency reliability (alpha $>0.80$ ) and factorial validity. ${ }^{39}$ The scale has seven items assessing HCPs' perspectives on the following intervention attributes: the appropriateness of the intervention for older rural patients and their families to manage warning signs of worsening health conditions, its effectiveness, risks and ease of use. ${ }^{39}{ }^{41}$ Five additional items will assess other aspects of acceptability, including HCPs' perspectives on the relevance and applicability of the intervention for this population, their confidence in providing it and the likelihood and frequency of using it in practice. ${ }^{40}$ A 5-point scale ranging from not at all (0) to very much (4) will be used in the rating of all items. The intervention will be considered acceptable if the attributes have an average (mean or median) rating greater than 2 (the midpoint of the response scale). ${ }^{40}$

With regard to demographics and professional profiles, a questionnaire on demographics (eg, age and gender) and professional profile (eg, years of experience and highest level of education) with standard questions previously used by our team will be used to describe the sample. ${ }^{40}$

\section{Qualitative semistructured focus group questions}

A qualitative semistructured interview guide will direct the focus group discussions to further explore HCPs' perspectives on the acceptability of a warning signs intervention, and the barriers and facilitators to providing it to older rural medical patients at risk of hospital readmission and their families (online supplementary appendix A). Questions, which will be guided by the ratings on the Intervention Acceptability Scale items, as well as the constructs of the intervention acceptability (eg, perceived effectiveness of the intervention) and knowledge-toaction (eg, perceived barriers) frameworks, will be pilot tested for clarity, comprehension and time commitment in one focus group prior to use in the full project. Questions will prompt HCPs to discuss why the intervention was rated as it was, and barriers and facilitators to its delivery to older rural medical patients at risk of readmission and their families.

\section{Procedures}

Consenting HCPs will be invited to participate in small telephone focus groups ( $\mathrm{n}=4-6 \mathrm{HCPs}) .{ }^{34}$ Telephone focus groups were selected to address logistical and geographic barriers to participation. We will mail to participating HCPs a package containing (1) the Intervention Acceptability Scale and the additional items assessing other aspects of intervention acceptability, which HCPs will have the option of completing online or in hard copy (based on preference) prior to the focus groups; (2) the questionnaire on demographics and professional data; and (3) the intervention logic model (online supplementary appendix B), which was synthesised from the empirical literature and our prior research, ${ }^{13} 18-2242$ and describes the warning signs intervention's goals, activities, mode of delivery, dose, anticipated benefits, and the human and material resources required to provide it. 
In collaboration with our knowledge users, we will review the intervention acceptability scores (average rating and range of values) and finalise the semi-tructured interview guides for each stratum. Then, telephone focus groups will be conducted at convenient times for HCPs by a research associate (RA) with doctoral preparation in qualitative methods and experience in conducting telephone focus groups. ${ }^{26}$ The RA will explore HCPs' perspectives on the overall acceptability of the intervention and then, for each stratum, summarise HPCs' acceptability ratings of the intervention. Acceptability ratings that will be summarised are those of the individual stratum (ie, nurses, allied healthcare professionals and physicians) participating in the focus group discussion, not the whole sample. Finally, the RA will further explore the acceptability ratings by engaging HCPs in a semistructured discussion on the acceptability of the intervention and barriers and facilitators to its delivery. Established interview strategies (eg, encouraging HCPs to respond to and build on each other's views and comments, reflecting convergent and divergent opinions back to the group, and turn-taking so that all voices are heard) will maximise group interaction and promote equal engagement by all participants. ${ }^{34}$ Focus groups will be approximately $60 \mathrm{~min}$ in length, audio-recorded and transcribed verbatim. Recruitment will be initiated by the knowledge users on our team and will involve introducing the study at staff meetings, posting flyers at the hospital sites, and raising awareness of the study via email and on social media. Approaches to promote participation include holding the focus groups during non-working hours via telephone, providing a $\$ 75$ gift card, ${ }^{43}$ asking HCPs to refer colleagues $^{44}$ and conducting 1:1 interviews with HCPs who are unable to attend a focus group. ${ }^{45}$

\section{Data analysis plan}

Descriptive statistics in accordance with each variable's level of measurement will be used to describe HCPs' demographic and professional profiles (eg, mean for age and frequencies for gender) and their rating of the intervention's acceptability (eg, mean and SD). The dispersion (eg, IQR) of the acceptability data will also be examined to ensure that outlying values do not distort the mean. In such cases, the median will be reported. All quantitative data will be stored and analysed in the Statistical Package for the Social Sciences.

Conventional qualitative content analysis of the interview data, as described by Hsieh and Shannon, ${ }^{46}$ will be performed simultaneously with data collection (facilitated by NVivo V.12) and will centre on elucidating (1) why the intervention's acceptability was rated as it was on the items assessing intervention acceptability; and (2) facilitators and barriers to its delivery, within and across settings (hospital and community). According to Hseih and Shannon's formulation of conventional qualitative content analysis, coding categories will be derived inductively directly from the text data. Analysis will thus involve the development of preliminary codes and their organisation into hierarchical categories. Each code and category will be defined; the interconnections between them will be documented, and exemplars for each will be selected. Coding will be conducted independently by two members of the research team, and when there is disagreement among the coders as to the interpretation of particular aspects of participants' narratives, these differences will be brought forward to the team and debated until 'intersubjective consensus' is achieved (Miles $e t$ al, p13). ${ }^{47}$ Data will be examined for patterns and discrepancies in HCPs' narratives (eg, by professional designation, region and gender) and acceptability item ratings using role-ordered and conceptually clustered matrices and analytical memoing. HCPs' perspectives will be summarised within each stratum and compared across strata and region to identify any patterns of similarity and difference. Strategies for trustworthiness will be employed throughout the research process. Confirmability will be ensured through the creation of an audit trail, while credibility will be ensured by having different research team members independently analyse the data and involving knowledge users in interpreting the findings. ${ }^{48}$ Dependability will be ensured through detailed methodological reporting and transferability through the presentation of the sample's demographic features (eg, gender and region of practice).$^{48}$ This context-sensitive approach will help illuminate how to tailor the implementation plan for the warning signs intervention to practice settings in rural Southwestern and Northeastern Ontario. Findings will enable the identification of commonalities in perspectives that are likely to be highly transferable beyond Ontario, as well as implementation issues (eg, lack of confidence in providing the intervention) and how these can be addressed to enable delivery of the intervention in rural communities. A list of barriers and facilitators to implementing the warning signs intervention, as identified by HCPs, will be created.

\section{PATIENT AND PUBLIC INVOLVEMENT}

Consultations with members of the public in the fall of 2018 confirmed the importance of strengthening the warning signs aspect of rural TC. The study team that evolved from these consultations includes a nurse practitioner, a geriatrician and family physician, a lay community member, and several hospital and community healthcare administrators. They constitute the knowledge users on our team. Because the study focuses on gathering HCPs' perspectives on an intervention previously identified by patients as critical to TC, a patient representative is not included.

The knowledge users on our team are deeply embedded in their rural communities, and they have played essential roles in supporting the development of the study to ensure its relevance to rural TC. They will continue to be actively involved (eg, helping to recruit HCPs, navigate the Ontario rural context, interpret findings, prepare lay summaries and recruitment scripts, and implement 
end-of-grant knowledge translation (KT)) as the study progresses.

To maximise collaboration and to ensure that knowledge users are invested in using the study findings, an integrated knowledge translation (i-KT) approach is embedded throughout this study. This approach employs a collaborative model of shared governance that promotes egalitarian dialogue and positions knowledge users as active members (eg, study objectives; questions and design were developed in partnership with them).$^{2430}$ As the research study unfolds, we will continuously draw on their expertise to refine and extend our KT plan. To maximise the relevance of the study's findings, knowledge users will be represented on an advisory committee that will guide the conduct of proposed activities, review progress and help interpret the findings. We will cocreate an i-KT evaluation plan to help us leverage facilitators to collaboration and mitigate potential barriers. ${ }^{49}$ To address gendered or professional power differentials that may carry over into our collaboration, we will structure meetings so that all members have opportunities to participate. Based on team members' communication preferences, a combination of quarterly videoconference and monthly email communication will be used.

Our end-of-grant KT objectives are (1) to disseminate findings on HCPs' perspectives on the acceptability of the intervention and the barriers and facilitators to its implementation and (2) to raise awareness of these findings. To achieve these objectives, dissemination strategies include communicating key findings to relevant audiences through plain language summary and policy briefs tailored to end users $\mathrm{s}^{50}$ and distributing the warning signs intervention logic model. These dissemination materials will be introduced to targeted knowledge users at separate interactive web-conference dialogues for policymakers and HCPs, as well as through conference presentations for researchers. The webinars will be posted on the York University Centre for Ageing Research \& Education website. Findings will be published in openaccess journals to make them available to national and international researchers and knowledge users, who can use the logic model and our approaches (eg, evaluating the acceptability of the warning signs intervention) as guidance in designing plans to implement the intervention in different rural communities. A press release of the findings will be distributed by York University's communications department.

\section{DISCUSSION}

High rates of hospital readmissions and emergency department visits indicate TC's failure to support older rural medical patients and their families at home in the postdischarge period, which is an urgent national and international concern. ${ }^{51} 52$ This study builds on our prior collaborations to illuminate HCPs' perspectives on a warning signs intervention and to build new knowledge on how to improve TC. It extends our prior
TC research by bringing an intervention, which rural patients and families rated highly acceptable, ${ }^{5}$ to HCPs and using a systematic process to assess their perspectives on its acceptability. This process will highlight potential implementation issues that need to be addressed to build HCPs' capacity to deliver the intervention with fidelity, ${ }^{53}$ thereby informing future studies and practice to improve the effectiveness of TC in rural communities for a key population at risk of adverse TC outcomes.

We anticipate that HCPs will view the warning signs intervention as relevant and applicable to their practice but will vary in their confidence providing it, and may encounter barriers to its delivery, such as lack of knowledge related to the warning signs intervention and poor communication among HCPs, patients and families. The findings may point to potential strategies, such as training HCPs on this warning signs intervention and incorporating tools for improving communication. Findings will also inform future evidence-based research and TC policy development to address rural-urban disparities in TC effectiveness, which is currently a major policy focus in Ontario and other jurisdictions. ${ }^{52} 54$

The intervention logic model, which will be disseminated in end-of-grant KT activities, is a useful tool to help HCPs understand how the intervention works, what they need to do and when, and the expected outcomes. The logic model can inform the implementation of the intervention by different HCPs in different settings, thereby facilitating communication among them and with patients and families. Overall, the study has great potential to promote more equitable services between communities at both regional and national levels. The inclusion of researchers from other parts of Canada will support future scaling of the intervention at a national level. Using multiple sites and providing detailed HCP demographic and professional data will enhance the transferability of findings to similar settings in Ontario and Canada.

\section{Author affiliations}

${ }^{1}$ School of Nursing, York University, Toronto, Ontario, Canada

2Daphne Cockwell School of Nursing, Ryerson University, Toronto, Ontario, Canada ${ }^{3}$ School of Rehabilitation Science, McMaster University, Hamilton, Ontario, Canada ${ }^{4}$ Occupational Science and Occupational Therapy, University of Toronto, Toronto, Ontario, Canada

${ }^{5}$ Faculty of Nursing, University of Alberta, Edmonton, Alberta, Canada

${ }^{6}$ Trent School of the Environment, Trent University, Peterborough, Ontario, Canada

Acknowledgements We thank the anonymous Canadian Institutes of Health Research peer reviewers and the knowledge users for their contribution to the study.

Contributors All authors made substantial contributions to the conception or design of the protocol, drafted or critically revised the protocol for important intellectual content, approved the final version of the protocol and agreed to be accountable for all aspects of the work in ensuring that questions related to the accuracy or integrity of any part of the work are appropriately investigated and resolved. Specifically, MTF led consultations with knowledge users on the conception of the protocol. MTF and JIB led the overall writing of the protocol. SS contributed her expertise on intervention acceptability assessment and assisted in devising the data collection tools. MWS and BN-K contributed their expertise on rural research. ED contributed her expertise on qualitative research. SD contributed her expertise on interprofessional practice and qualitative methods. JY contributed her expertise on implementation science. 
Funding This work is supported by the Canadian Institutes of Health Research (funding reference number 163072). The Canadian Institutes of Health Research had no role in the design of the protocol or in the writing of this manuscript.

Competing interests None declared.

Patient and public involvement Patients and/or the public were involved in the design, conduct, reporting or dissemination plans of this research. Refer to the Design section for further details.

Patient consent for publication Not required.

Ethics approval Ethics approval has been obtained from the Research Ethics Board at York University (certificate number e219-241). Written informed consent will be obtained from all participants and, as per our ethics protocol, hard copy data (eg, consent forms, questionnaires, interview transcripts and fieldnotes) will be stored in a locked filing cabinet in the principal investigator's office at York University for 10 years after study completion. Electronic data will be stored on a dedicated, encrypted, password-protected hard drive

Provenance and peer review Not commissioned; externally peer reviewed.

Open access This is an open access article distributed in accordance with the Creative Commons Attribution Non Commercial (CC BY-NC 4.0) license, which permits others to distribute, remix, adapt, build upon this work non-commercially, and license their derivative works on different terms, provided the original work is properly cited, appropriate credit is given, any changes made indicated, and the use is non-commercial. See: http://creativecommons.org/licenses/by-nc/4.0/.

ORCID iDs

Mary T Fox http://orcid.org/0000-0003-0572-3892

Jeffrey I Butler http://orcid.org/0000-0003-2765-7208

Souraya Sidani http://orcid.org/0000-0002-9115-2389

Evelyne Durocher http://orcid.org/0000-0002-2122-3997

Behdin Nowrouzi-Kia http://orcid.org/0000-0002-5586-4282

Sherry Dahlke http://orcid.org/0000-0001-6599-3101

Mark W Skinner http://orcid.org/0000-0001-7148-6827

\section{REFERENCES}

1 Coleman EA, Smith JD, Frank JC, et al. Preparing patients and caregivers to participate in care delivered across settings: the care transitions intervention. J Am Geriatr Soc 2004;52:1817-25.

2 Gibson MJ, Kelly KA, Kaplan AK. Family caregiving and transitional care: a critical review. family care giver alliance, National centre on care giving. San Francisco, CA, 2012.

3 Kulig JC, Williams AM. Health in rural Canada. Vancouver, BC: UBC Press, 2011.

4 Canadian Institute for Health Information. All-Cause readmission to acute care and return to the emergency department. Ottawa, Ontario: Canadian Institute for Health Information, 2012.

5 Fox MT, Sidani S. Final report submitted to the Ontario Ministry of health and long-term care: adapting hospital-to-home transitional care interventions to the Ontario rural healthcare context. Toronto, Ontario, 2019.

6 Herron R, Skinner M. Rural spaces and places of health and health care. In: Crooks V, Andrews JG, Pearce J, eds. Handbook of Health Geography. London (UK): Routledge International Handbook Series, 2018: 267-72.

7 Kenning C, Coventry PA, Bower P. Self-Management interventions in patients with long-term conditions: a structured review of approaches to reporting inclusion, assessment, and outcomes in multimorbidity. J Comorb 2014;4:37-45.

8 Roberts KC, Rao DP, Bennett TL, et al. Prevalence and patterns of chronic disease multimorbidity and associated determinants in Canada. Health Promot Chronic Dis Prev Can 2015;35:87-94.

9 Reinhard SR, Levine C, Samis S. Home alone: family caregivers providing complex chronic care. Washington, DC: AARP Public Policy Institute and the United Hospital Fund, 2012.

10 Health Quality Ontario. Quality Monitor: 2012 Report on Ontario's Health System. Toronto, Ontario, 2012.

11 Grant K, Church E. No place like home? Investigating Ontario's home-care shortcomings, in The Globe and Mail. Toronto, Ontario, 2015.

12 Jay Biem $\mathrm{H}$, Hadjistavropoulos $\mathrm{H}$, Morgan D, et al. Breaks in continuity of care and the rural senior transferred for medical care under regionalisation. Int $J$ Integr Care 2003;3:e03.

13 Fox MT, Persaud M, Maimets I, et al. Effectiveness of early discharge planning in acutely ill or injured hospitalized older adults: a systematic review and meta-analysis. BMC Geriatr 2013;13:70.
14 Boston University Medical Center. Project red Re-engineered discharge, 2013. Available: http://www.bu.edu/fammed/projectred/ index.html [Accessed Sep 2019].

15 Jackson CT, Trygstad TK, DeWalt DA, et al. Transitional care cut hospital readmissions for North Carolina Medicaid patients with complex chronic conditions. Health Aff 2013;32:1407-15.

16 Naylor MD, Brooten D, Campbell R, et al. Comprehensive discharge planning and home follow-up of hospitalized elders. JAMA 1999;281:613-20.

17 Naylor MD, Brooten DA, Campbell RL, et al. Transitional care of older adults hospitalized with heart failure: a randomized, controlled trial. $J$ Am Geriatr Soc 2004;52:675-84.

18 Allen J, Hutchinson AM, Brown R, et al. Quality care outcomes following transitional care interventions for older people from hospital to home: a systematic review. BMC Health Serv Res 2014;14:1-27.

19 Legrain S, Tubach F, Bonnet-Zamponi D, et al. A new multimodal geriatric discharge-planning intervention to prevent emergency visits and rehospitalizations of older adults: the optimization of medication in aged multicenter randomized controlled trial. J Am Geriatr Soc 2011;59:2017-28.

20 Wee S-L, Loke C-K, Liang C, et al. Effectiveness of a national transitional care program in reducing acute care use. J Am Geriatr Soc 2014:62:747-53.

21 Coleman EA, Parry C, Chalmers S, et al. The care transitions intervention: results of a randomized controlled trial. Arch Intern Med 2006;166:1822-8.

22 Parry C, Min S-J, Chugh A, et al. Further application of the care transitions intervention: results of a randomized controlled trial conducted in a fee-for-service setting. Home Health Care Serv Q 2009;28:84-99.

23 Proctor E, Silmere H, Raghavan R, et al. Outcomes for implementation research: conceptual distinctions, measurement challenges, and research agenda. Adm Policy Ment Health 2011;38:65-76.

24 Sekhon M, Cartwright M, Francis JJ. Acceptability of healthcare interventions: an overview of reviews and development of a theoretical framework. BMC Health Serv Res 2017;17:1-13.

25 Campbell NC, Murray E, Darbyshire J, et al. Designing and evaluating complex interventions to improve health care. BMJ 2007;334:455-9.

26 Fox MT, Butler JI. Nurses' perspectives on how operational leaders influence function-focused care for hospitalised older people. J Nurs Manag 2016;24:1119-29.

27 Hirschman KB, Shaid B, Bixby MB, et al. Transitional care in the patient-centered medical home: lessons in adaptation. $J$ Healthc Qual 2017;39:67-77.

28 Kim CS, Flanders SA. Transitions of care. Ann Intern Med 2013;158:ITC3-1.

29 Scott AM, Li J, Oyewole-Eletu S, et al. Understanding facilitators and barriers to care transitions: insights from project achieve site visits. Jt Comm J Qual Patient Saf 2017;43:433-47.

30 Graham ID, Logan J, Harrison MB, et al. Lost in knowledge translation: time for a MAP? J Contin Educ Health Prof 2006;26:13-24.

31 Tchameni Ngamo S, Souffez K, Lord C, et al. Do knowledge translation (KT) plans help to structure KT practices? Health Res Policy Sys 2016;14:46.

32 Statistics Canada. Population, urban and rural, by Province and territory. Ottawa, Ontario: Statistics Canada, 2011. https://www150. statcan.gc.ca/n1/pub/11-630-x/11-630-x2015004-eng.htm

33 Segeo JA. Work group culture, stress, and hostility: correlations with organizational outcomes. J Nurs Adm 1996;26:39-47.

34 Krueger RA, Casey MA. Focus groups: a practical guide for applied research. 5th edn. Thousand Oaks, CA: Sage Publications, 2014.

35 Dahlke SA, Phinney A, Hall WA, et al. Orchestrating care: nursing practice with hospitalised older adults. Int J Older People Nurs 2015;10:252-62.

36 Morse JM, Field PA. Qualitative research methods for health professionals. 2nd ed. Thousand Oaks: Sage, 1995.

37 Carlsen B, Glenton C. What about N? A methodological study of sample-size reporting in focus group studies. BMC Med Res Methodol 2011;11:1-10.

38 Norman GR, Streiner DL. Biostatistics: the bare essentials. 4th edn. Hamilton, Ontario, Canada: B.C. Decker, 2013.

39 Sidani S, Epstein DR, Fox M, et al. Psychometric properties of the treatment perception and preferences measure. Clin Nurs Res 2018;27:743-61.

40 Sidani S, Manojlovich M, Doran D, et al. Nurses' perceptions of interventions for the management of patient-oriented outcomes. Worldviews Evid Based Nurs 2016;13:66-74. 
41 Sidani S, Epstein DR, Bootzin RR, et al. Assessment of preferences for treatment: validation of a measure. Res Nurs Health 2009;32:419-31.

42 Fox MT, Sidani S, Butler JI, et al. Protocol of a multimethod descriptive study: adapting hospital-to-home transitional care interventions to the rural healthcare context in Ontario, Canada. BMJ Open 2019;9:e028050.

43 Mohan D, Rosengart MR, Fischhoff B, et al. Using incentives to recruit physicians into behavioral trials: lessons learned from four studies. BMC Res Notes 2017;10:776.

44 Fulda KG, Hahn KA, Young RA, et al. Recruiting practice-based research network (PBRN) physicians to be research participants: lessons learned from the North Texas (NorTex) needs assessment study. J Am Board Fam Med 2011;24:610-5.

45 Robitaille $\mathrm{H}$, Légaré $\mathrm{F}$, Tre $\mathrm{G}$. A systematic process for recruiting physician-patient dyads in practice-based research networks (PBRNs). J Am Board Fam Med 2014;27:740-9.

46 Hsieh H-F, Shannon SE. Three approaches to qualitative content analysis. Qual Health Res 2005;15:1277-88.

47 Miles MB, Huberman AM, Saldana J. Qualitative data analysis. Los Angeles, CA: Sage, 2013.

48 Shenton AK. Strategies for ensuring trustworthiness in qualitative research projects. EFI 2004;22:63-75.
49 Parry D, Salsberg J, Maccauley AC. A guide to researcher and knowledge-user collaboration in health research, 2014. Available: http://www.cihr-irsc.gc.ca/e/44954.html [Accessed Sep 2019].

50 Cunningham SD, Card JJ. Realities of replication: implementation of evidence-based interventions for HIV prevention in real-world settings. Implementation Sci 2014;9:5-13.

51 Donner G, McReynolds J, Smith K, et al. Bringing care home. Report of the expert group on home and community care, 2015. Available: http://health.gov.on.ca/en/public/programs/ccac/docs/hcc_report. pdf [Accessed Sep 2019].

52 Kansagara D, Chiovaro JC, Kagen D, et al. Transitions of care from hospital to home: an overview of systematic reviews and recommendations for improving transitional care in the Veterans health administration. Washington (DC, 2015.

53 Sidani S, Braden CJ. Design, evaluation, and translation of nursing interventions. Oxford (UK: Wiley-Blackwell, 2011.

54 Health Quality Ontario. Adopting a common approach to transitional care planning: helping health links improve transitional and coordination of care. Ottawa, Ontario, 2014. https://www.google. $\mathrm{com} /$ url? sa $=\mathrm{t} \& \mathrm{rct}=\mathrm{j} \& \mathrm{q}=\&$ esrc $=\mathrm{s} \&$ source $=$ web\&cd $=1 \& \mathrm{cad}=\mathrm{rja} \&$ uact $=8 \&$ ved $=2$ ahUKEwiZyqbW1fvkAhUNvJ4KHZAwD2IQFjAAegQ IAXAC\&url=http\%3A\%2F\%2Fwww.hqontario.ca\%2FPortals\%2F0\% 2Fdocuments\%2Fqi\%2Fhealth-links\%2Fbp-improve-packagetraditional-care-planning-en.pdf\&usg=AOvVaw2pLj2KS6pv5Jf6 AsqTCFgz 